\title{
Publisher Correction: Safety and feasibility of CRISPR-edited T cells in patients with refractory non-small-cell lung cancer
}

You Lu (D), Jianxin Xue, Tao Deng, Xiaojuan Zhou, Kun Yu, Lei Deng, Meijuan Huang, Xin Yi, Maozhi Liang, Yu Wang, Haige Shen, Ruizhan Tong, Wenbo Wang, Li Li, Jin Song, Jing Li, Xiaoxing Su, Zhenyu Ding, Youling Gong, Jiang Zhu, Yongsheng Wang, Bingwen Zou, Yan Zhang, Yanying Li, Lin Zhou, Yongmei Liu, Min Yu, Yuqi Wang, Xuanwei Zhang, Limei Yin, Xuefeng Xia, Yong Zeng, Qiao Zhou, Binwu Ying, Chong Chen, Yuquan Wei, Weimin Li and Tony Mok

Correction to: Nature Medicine https://doi.org/10.1038/s41591-020-0840-5, published online 27 April 2020.

In the version of this article initially published, the label 'TPS $1 \geq 1 \%$ ' in the top left box of Fig. 1a was incorrect. The correct label is 'TPS $\geq 1 \%$ '. The error has been corrected in the HTML and PDF versions of the article.

Published online: 18 June 2020

https://doi.org/10.1038/s41591-020-0973-6

๑ The Author(s), under exclusive licence to Springer Nature America, Inc. 2020

\section{Publisher Correction: China needs a national intelligent syndromic surveillance system}

Peng Jia (iD and Shujuan Yang

Correction to: Nature Medicine https://doi.org/10.1038/s41591-020-0921-5, published online 20 May 2020.

In the version of this article initially published, the numbering and order of the second and third affiliations $\left({ }^{2}\right.$ West China School of Public Health and West China Fourth Hospital, Sichuan University, Chengdu, China. ${ }^{3}$ International Initiative on Spatial Lifecourse Epidemiology, Hong Kong, China.) was incorrect. The correct order is as follows: ${ }^{2}$ International Initiative on Spatial Lifecourse Epidemiology, Hong Kong, China. ${ }^{3}$ West China School of Public Health and West China Fourth Hospital, Sichuan University, Chengdu, China. Also, the second half of the first time frame ("under 2 days") in the first sentence of the third paragraph was incorrect. The correct phrasing is "under 2 hours." The errors have been corrected in the HTML and PDF versions of the article.

Published online: 18 June 2020

https://doi.org/10.1038/s41591-020-0977-2

(c) The Author(s), under exclusive licence to Springer Nature America, Inc. 2020

\section{Addendum: Estimating clinical severity of COVID-19 from the transmission dynamics in Wuhan, China}

Joseph T. Wu (D), Kathy Leung, Mary Bushman, Nishant Kishore, Rene Niehus, Pablo M. de Salazar, Benjamin J. Cowling (D, Marc Lipsitch and Gabriel M. Leung

Addendum to: Nature Medicine https://doi.org/10.1038/s41591-020-0822-7; published online 19 March 2020

On 17 April 2020, the Wuhan Municipal Health Commission revised the official COVID-19 death counts in Wuhan up by 1,290 to 3,869. No new information on the age distribution of COVID-19 deaths in China has been published since February 2020. In light of the changes in the number of fatalities in Wuhan, we are providing a revised estimate of clinical severity as follows, assuming that the age distribution of COVID-19 deaths remains the same as before.

We assumed that the abovementioned revision of the total number of COVID-19 deaths in Wuhan would increase the number of confirmed COVID-19 deaths during our study period by the same proportion; i.e., from 2,169 to $2,169 \times 3,869 /(3,869-1,290)=3,254$. The resulting age-specific symptomatic case-fatality risks (sCFRs) are shown in Fig. 1 in this Addendum. The overall sCFR would increase to $2.2 \%$ (95\% credible interval, $1.4-3.2 \%), 2.0 \%(1.2-3.4 \%)$ or $1.8 \%(1.0-2.8 \%)$ if the probability of developing symptoms after infection $\left(P_{\text {sym }}\right)$ was $0.5,0.75$ or 0.95 , respectively. If $P_{\text {sym }}=0.5$, the sCFRs are $0.5 \%(0.2-1.0 \%), 0.8 \%(0.5-1.2 \%)$ and $3.9 \%(2.6-5.9 \%)$ for those $<30$ years of age, $30-59$ years of age and $>59$ years of age, respectively.

We will update our estimates again as and when new and more detailed information, including the total number and age distribution of COVID-19 deaths, are made available. 

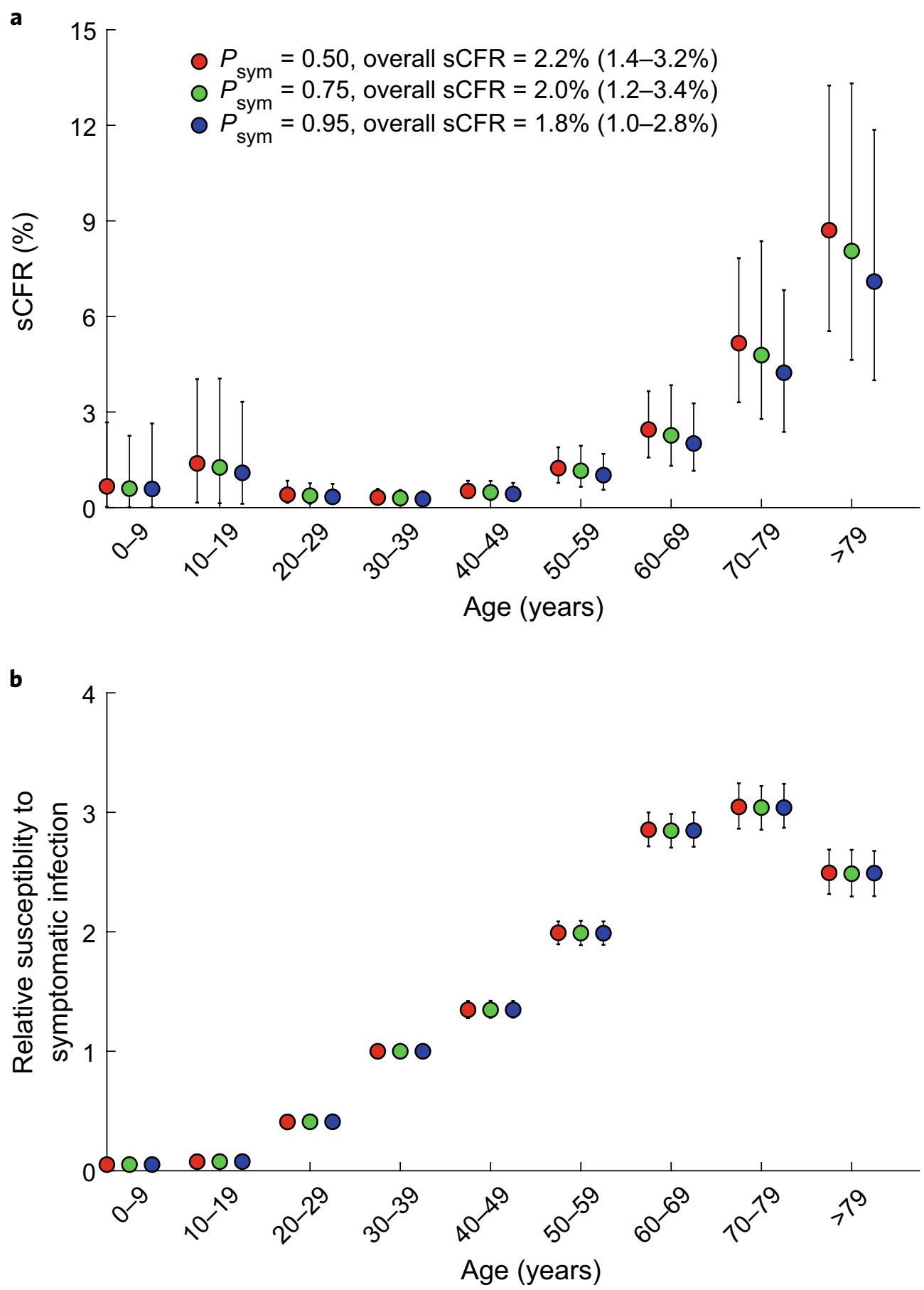

Fig. 1 | Estimates of age-specific SCFR values and susceptibility to symptomatic infection for COVID-19 in Wuhan. a, Estimates of age-specific sCFRs, assuming $P_{\text {sym }}$ is 0.5 (red), 0.75 (green) or 0.95 (blue). b. Estimate of relative susceptibility to symptomatic infection by age, assuming $P_{\text {sym }}$ is 0.50 (red), 0.75 (green) or 0.95 (blue). Symbols in a,b show the posterior means, and the bars show $95 \%$ credible intervals.

Published online: 21 May 2020

https://doi.org/10.1038/s41591-020-0920-6

(C) The Author(s), under exclusive licence to Springer Nature America, Inc. 2020 